\title{
DOS VERSIONES DE PSICOLOGÍA FENOMENOLÓGICA. EN TORNO A LA INFLUENCIA DE WILLIAM JAMES EN LAS INVESTIGACIONES LÓGICAS DE EDMUND HUSSERL
}

\author{
Raúl E. Zegarra Medina
}

Pontificia Universidad Católica del Perú

El artículo constituye una breve investigación histórica y teórica en torno a los principales nexos entre el pensamiento temprano de William James y el trabajo desplegado por Edmund Husserl en las Investigaciones lógicas. A través de un examen preliminar de las relaciones personales entre ambos autores, pasaremos a un estudio sobre el aparato conceptual desarrollado por James, sobre todo en Principios de psicología, con el objetivo de contrastarlo con el planteado por Husserl, mostrando cómo el primer autor esbozó, entre otros, los conceptos fenomenológicos de intencionalidad y objetividad ideal.

Palabras clave:

William James, Edmund Husserl, fenomenología, intencionalidad, psicología 
El siguiente trabajo presenta lo que podríamos llamar un esfuerzo por hacer historia de los conceptos, historia de la filosofía. Mi interés, básicamente, es mostrar cuáles son las relaciones filosóficas, tanto verificables como posibles, de dos de las más importantes obras del pensamiento occidental reciente. Por un lado, los Principios de psicología (PP, 1890) de William James y, por el otro, las Investigaciones lógicas (IL, 1900-190I/1913) de Edmund Husserl. Con esa finalidad, dividiré el trabajo en tres secciones. La primera de ellas consistirá en una exposición de las principales relaciones histórico-filosóficas entre ambos autores. En ese apartado veremos cuáles fueron los vínculos personales e intelectuales que mantuvieron James y Husserl y examinaremos las razones por las cuales es posible sostener que el peso de James en las primeras reflexiones del filósofo alemán fue fundamental. La segunda parte del texto se dedicará, sobre todo, al estudio de algunos pasajes de los PP, los mismos que se pondrán en relación con otros de las IL para que pueda verse, a través del examen de los propios textos, si la hipótesis de la influencia jamesiana en el pensamiento temprano de Husserl es válida o no. En vista de que se trata de dos obras de gran envergadura, me concentraré básicamente en el análisis que ambos autores hacen de la intencionalidad, aunque las conexiones pueden establecerse allende ese contexto. Finalmente, en un tercer momento, haré un balance breve de las dos primeras partes del trabajo sugiriendo algunas conclusiones en torno a la relación entre James y Husserl.

\section{§ 1. El prejuicio vs. la admiración: una relación dispareja}

Sobre la relación entre James y Husserl no existe abundante información, pero sí la suficiente como para hacernos una idea más o menos clara del tipo de vínculos que enlazaron a ambos autores. La primera cuestión es meramente cronológica, aunque relevante: James y Husserl eran coetáneos. Más aun y, esto es lo relevante, ambos escribieron obras que cambiaron el curso de la reflexión de su época para sus respectivos ámbitos de competencia. Como se indicó más arriba, James publicó en 1890 sus PP; Husserl presentó su primera edición de las IL apenas un decenio después. No solo se trata de pensadores que vivieron en épocas paralelas, sino que publicaron obras determinantes en esferas de tiempo muy cercanas. Sin embargo, las diferencias en el trato mutuo de las obras se hacen patentes sin mucha dificultad. 
Husserl leyó con mucho interés a James, mientras que, todo así lo indica, James nunca leyó a Husserl. Existen varias explicaciones para esta situación. La primera refiere a circunstancias indirectas: James murió en 1910 y Husserl más de 20 años después. Para la época en que aparecieron las $I L$, entonces, solo nueve años alejaban al primer autor de su muerte $y$, en toda esa etapa previa James estuvo inmerso en una gran producción intelectual tanto por escrito como por medio de conferencias públicas para la difusión de su pragmatismo. En ese sentido, es comprensible que la obra de Husserl no fuese una de sus prioridades, sobre todo porque ya para esa época él había dejado un poco de lado sus investigaciones psicológicas para concentrarse en los desarrollos de importantes obras como Pragmatism (1907), A Pluralistic Universe (1909) y los póstumos Essays in Radical Empiricism (1912).

Si bien esto puede dar un cierto marco explicativo, existe otra razón por la cual James no leyó las IL de Husserl, aunque no hay seguridad de si esta corresponde a un hecho verificable. En todo caso, el rumor existe y el rumor llegó a los oídos de Husserl, quien lo tomó, evidentemente, como una ofensa: "Walter B. Pitkin has claimed that James turned the publishing house of Mifflin against Husserl and thus obstructed the publication in America of Pitkin's translation of the Logical Investigations. It is true that James distanced himself from contemporary Continental European logic. But it is clear that he had not seen either a copy of the second volume of the Logical Investigations, or Pitkin's translation of the Prolegomena. Moreover, there were no books by Husserl in James's personal library"'. Richard Cobb-Stevens narra el mismo suceso en la entrada "William James" de la Encyclopaedia of Phenomenology: "At the height of his career William James (1842-1910) unwittingly made a decision that in effect deprived several generations of philosophers in the United States of easy access to one of the greatest philosophical works of the $20^{\text {th }}$ century, Edmund Husserl's Logische Untersuchungen (1900-190I). In response to an inquiry from a prestigious American publishing house about the merits of an already completed translation of Husserl's work, James advised against its publication on the grounds that no one in America would be interested in another German work on logic. The cavalier and chauvinistic tone of this

\footnotetext{
' Herzog, M., "William James and the Development of Phenomenological Psychology in Europe", en: History of the Human Sciences, VIII, No. I (1995), p. 32.
} 
remark is untypical and unworthy of James ${ }^{2}$, who would no doubt have been chagrined to discover that in the second edition of his work Husserl generously refers to James's 'genius for observation in the field of descriptive psychology'3, and expresses his indebtedness to James for his help in achieving 'my release from the psychologistic position"'4.

Como puede verse, parece que una importante cuota de prejuicio fue uno de los factores que determinó el desinterés de James. Prejuicio y desinterés tan graves como para lograr que las IL de Husserl no llegaran a la academia americana en una versión inglesa completa sino hasta 1970, mediante la traducción, de la segunda edición, realizada por J.N. Findlay ${ }^{5}$. Independientemente de que el rumor sea cierto o no -Spiegelberg no da fe de la versión de Pitkin, aunque sí acepta el prejuicio jamesiano contra los lógicos alemanes en sus últimos años ${ }^{6}$ lo cierto es que Pitkin comunicó esa información a Husserl y, a partir de ese momento, Husserl tomó distancia de James y ya no se refirió más a él en sus textos. La ofensa es totalmente comprensible, aunque es muy difícil saber si el hecho efectivamente aconteció. Lo interesante de esto es que nos conduce a examinar la historia desde el otro lado del Atlántico. ¿Qué cosas sí dijo Husserl de James antes de este incidente? Pasemos a revisar la información que tenemos al respecto.

Según indica el propio Husserl en unas notas personales de 1906, su primera lectura de James se llevó a cabo entre 189I y 1892. En ese momento, Husserl

\footnotetext{
${ }^{2}$ Como se desprende de sus intercambios epistolares, así como de la información que nos ofrecen sus biógrafos. Cf. James, H. (ed.), The Letters of William James, Boston: The Atlantic Monthly Press, 2v., 1920. Aunque existen, al menos, tres biografías importantes además de las que refiero a continuación, remito a estas por ser las que mejor conozco: Perry, R.B., The Thought and Character of William James, Nashville: Vanderbilt University Press, 1996 y Richardson, R.D., William James: In the Maelstrom of American Modernism, Nueva York: Mariner Books, 2006. La segunda obra tiene la ventaja de ser el trabajo más reciente sobre James en términos biográficos y que, por ende, presupone a los anteriores. Además, Richardson es un galardonado especialista en la realización de biografías, entre las cuales se destacan las dedicadas a Emerson (quien, además, fuese el padrino de William James) y Thoreau.

${ }^{3}$ La referencia exacta de lo dicho por Husserl será examinada en breve.

${ }^{4}$ Embree, L. y otros (eds.), Encyclopaedia of Phenomenology, Dordrecht/Boston/Londres: Kluwer Academic Publishers, 1997, p. 363.

${ }^{5}$ Cf. Husserl.net, consulta: 15 de junio de $2010<$ http://www.husserl.net/books/title. php?opt $=\mid$ \& source $=20>$.

${ }^{6}$ Cf. Spiegelberg, H., The Phenomenological Movement, La Haya: Nijhoff, 1984, p. 100 (nota); citado en: Herzog, M., op. cit., p. 32.
} 
era Privatdozent y se encontraba dando un curso sobre psicología en Halle, y fue en ese contexto en el que los PP de James llegaron a sus manos, según el propio Husserl indica, por sugerencia de Carl Stumpf ${ }^{7}$, el mejor amigo de James en la academia alemana. Husserl escribe lo siguiente el 25 de septiembre: "Then came the lectures on psychology in 1891/92, which gave me occasion to examine the writings of descriptive psychology, longingly anticipated. James' psychology, of which I could only read a few pieces and very little at that, provided a few flashes. I saw how an acute and original man let himself be bound by no tradition; and how he sought to grasp and describe that which he contemplated. This influence was certainly not without its effect upon me, although I was only able to read and understand a few pages. Yes, to describe and to be exact, that was extremely necessary. But I did not read and make notes upon larger sections of his writings until after the appearance of my treatise of $1894^{8} "$. Como se ve, se trata de una influencia declarada por el mismo Husserl y cuyas peculiaridades trataremos de examinar más adelante. Ahora bien, es importante notar que no se trató de una lectura superficial de la psicología de James ${ }^{10}$ : si bien, al inicio, el filósofo afirma que solo revisó de modo escueto al autor de Pragmatismo, lo cierto es que más tarde se dedicaría a un estudio riguroso de los PP, lo mismo que puede verse por la cantidad de apuntes que realizó en su propio ejemplar del libro, el cual se conserva en los Archivos Husserl".

\footnotetext{
${ }^{7}$ Cf. Crowell, S. (ed.), The New Yearbook for Phenomenology and Phenomenological Philosophy, Seattle: Noesis, 200I, p. 322. Husserl le habría confirmado esto a Dorion Cairns en 193I.

${ }^{8}$ Se refiere a sus "Psychologische Studien zur elementaren Logik", publicados en la revista Philosophische Monatshefte, No. 30.

${ }^{9}$ Herzog, M., op. cit., p. 3I. El texto original fue editado por Walter Biemel y publicado bajo el título de "Persönliche Aufzeichnungen", en: Philosophy and Phenomenological Research, XVI, No. 3 (1956), pp. 293-302. La misma cita se consigna, aunque en una traducción algo distinta, en Crowell, S., op. cit., pp. 32I-322. Puede accederse al fragmento en el idioma original y en el contexto de la edición de la obra completa del fenomenólogo alemán en Husserl, E., Einleitung in die Logik und Erkenntnistheorie. Husserliana XXIV, conferencias de 1906-1907 editadas por Ullrich Melle, Dordrecht/Boston/Lancaster: Martinus Nijhoff, 1984, Apéndice B IX, p. 443.

${ }^{10}$ Husserl tiene en mente los $P P$, aunque en el fragmento no se indique explícitamente.

"Husserl leyó los PP con gran intensidad. Colocó numerosas notas marginales con particular énfasis en los siguientes capítulos: Habit (IV), The Automaton-Theory (V), The Mind-Stuff Theory (VI), Methods and Snares of Psychology (VII), The Relation of Mind to Other Things (VIII), The Stream of Thought (IX), Attention (XI), Conception (XII), The Perception of Space $(X X)$, The Perception of Reality $(X X I)$, Reasoning $(X X I I)$ y Will $(X X V I)$. Cf. Crowell, S., op. cit., p. 321, nota 9 .
} 
Sin embargo, esa no es la única mención de James hecha por Husserl. En las mismas IL, nuestro autor se refiere a James en dos momentos. La primera es una alusión casi al paso; la segunda, sin embargo, es de mayor relevancia. Así, indica: "Las observaciones geniales de James en el terreno de la psicología descriptiva de las vivencias representativas no conducen forzosamente al psicologismo; como se ve en esta obra, puesto que las sugestiones que al notable investigador debo yo en el análisis descriptivo no han hecho más que favorecer mi abandono del punto de vista psicologista"'2. La cita aparece en el contexto de una nota al pie a unos comentarios sobre la teoría de Cornelius realizados por Husserl en el Apéndice a la Segunda Investigación Lógica, "La teoría de Hume en sus partidarios modernos”. Independientemente del contenido particular del debate con Cornelius, lo relevante del pasaje citado es que muestra una de las pocas menciones textuales explícitas de James hechas por Husserl. En la cita se puede ver no solo la generosidad y admiración que el filósofo alemán sentía por James, sino el valor que le daba a su psicología descriptiva como una importante entrada a la fenomenología que el propio Husserl desarrollaría.

Otra mención pequeña, pero significativa, se consigna en el prefacio a sus conferencias sobre "The Principal Aspects of Phenomenology and the Theory of Knowledge”, impartidas en el semestre de invierno de 1905-1906 en la Universidad de Göttingen. Allí, Husserl se refiere al autor de los PP en los siguientes términos: "James, whom I have studied and whose work has greatly inspired me”|3. Más allá de eso, y contrariamente al caso de James, Husserl había leído

\footnotetext{
${ }^{12}$ Husserl, E., Investigaciones lógicas, traducción de Manuel García Morente y José Gaos, 2da. edición, Madrid: Revista de Occidente, 1976, p. 37I, nota I6; en adelante, IL.

${ }^{13}$ Herzog, M., op. cit., p. 32. La cita corresponde a Husserl, E., Zur Phänomenologie des inneren Zeitbewusstseins, La Haya: Nijhoff, 1969, p. xvi. En su introducción a la traducción inglesa de la Husserliana X (la traducción del volumen que acabamos de mencionar), John Barnett Brough ofrece más información, aunque algo distinta, de la vertida por Herzog. En primer lugar, contrariamente a Herzog, Brough indica que las conferencias se impartieron en el semestre de invierno de la Universidad de Göttingen, pero el de 1904-1905. El texto de las mismas fue un hallazgo de Edith Stein en 1917, texto que en su versión original se encontraba dentro de un manojo de numerosas hojas, en muy mal estado, constituido por notas de Husserl que iban desde I90I en adelante. Otro dato relevante es que estas conferencias sobre "The Principal Aspects of Phenomenology and the Theory of Knowledge" (la traducción de Brough es ligeramente diferente) no se encuentran consignadas en la Husserliana $X$, por lo que, cabe conjeturar, la cita sobre James presentada por Herzog corresponde a un extracto de los mismos manuscritos $y$ debe haber sido recogida en la introducción por el editor alemán de dicha Husserliana, Rudolf Boehm, en 1966. Para más información, puede verse: Husserl, E., On the Phenomenology of the
} 
y tenía en su biblioteca personal casi toda la obra del autor estadounidense ${ }^{14}$, aunque para quien conoce la misma, se podría suponer que Husserl se sentía más cercano a los textos tempranos de James sobre psicología y no tanto a los desarrollos posteriores de su pragmatismo ${ }^{15}$.

En resumen, podríamos decir que la relación entre Husserl y James fue, básicamente, unidireccional: la atención vino desde Europa hacia los Estados Unidos y la reciprocidad fue prácticamente inexistente ${ }^{16}$. Las razones de esto han sido ya esbozadas, aunque, lamentablemente, parece que el peso del prejuicio fue una de las más importantes. Dicho esto, pasemos ahora a un estudio sobre los textos de nuestros autores para tratar de identificar los puntos de contacto filosófico más relevantes.

Consciousness of Internal Time (1893-1917), traducción, introducción y notas de J.B. Brough, Dordrecht/Boston/Londres: Kluwer Academic Publishers, 199I, p. xi y ss.

${ }^{14}$ Cf. Linschoten, J., Auf dem Weg zu einer phänomenologischen Psychologie, Berlín: de Gruyter, 1961, nota 72; citado en: Herzog, M., op. cit., p. 32.

${ }^{15}$ De hecho, como testimonia Herzog, en la última etapa del pensamiento de James se inicia el giro trascendental de la fenomenología de Husserl, el mismo que no solo lo terminó alejando del autor de los PP, sino de algunos de sus más importantes discípulos. El mismo Herzog consigna un dato interesante relativo a este distanciamiento cuyo punto más resaltante emerge frente a la figura de Josiah Royce. Un alumno estadounidense de Husserl, Winthrop Bell, confirmó en una carta personal a Spiegelberg (op. cit., p. 128, nota) que Husserl leyó intensamente los textos de Royce durante la época en que Bell estuvo en Göttingen (I9II-1914). No solo eso, sino que durante esos años, Husserl identificó el pensamiento tardío de James como una reacción crítica poco fundada a la filosofía de Royce en un nivel tal que la disertación doctoral de Bell, que recogía las reservas de James en torno a Royce, recibió críticas de Husserl por no haber leído y considerado con suficiente respeto al último autor. Como es sabido, más allá de la interpretación de Husserl, James se declaraba como un pensador crítico de la filosofía idealista del autor de The Religious Aspect of Philosophy (1885). Para más detalles, puede verse Herzog, M., op. cit., p. 43ss. Para información sobre la relación de profunda amistad y severa divergencia intelectual entre James y Royce, pueden verse las biografías de James citadas. También es útil la entrada "Josiah Royce" de la Stanford Encyclopedia of Philosophy (consulta: 14 de julio de 2010, <http:// plato.stanford.edu/entries/royce/\#Bio >).

${ }^{16}$ No tengo noticia de alguna comunicación directa dirigida por James a Husserl, aunque Linschoten afirma que James le envió una copia de "The Knowing of Things Together" (artículo aparecido en 1895 , fuera de tiraje en la época en que James hizo el envío y reeditado en The Meaning of Truth, en 1909, como parte del capítulo dos, "The Tigers in India") al filósofo alemán, la misma que fue leída por este último y que se conserva en el Archivo Husserl de la Universidad de Lovaina. De hecho, Herzog (op. cit., p. 32) sostiene que hay allí material relevante para identificar la influencia jamesiana en el concepto de intencionalidad en Husserl. Cabe suponer que el texto llegó a manos de Husserl vía, nuevamente, Carl Stumpf. 


\section{§ 2. Los Principios de Psicología, ¿una primera versión de la fenomenología de Husserl?}

Los primeros en apuntar la relación entre el pensamiento de Husserl y el de James fueron algunos importantes alumnos del filósofo alemán. Como es sabido, la Segunda Guerra Mundial trajo consigo una considerable migración de académicos europeos hacia los Estados Unidos y entre ellos se encontraron algunos exalumnos de Husserl, concretamente, Alfred Schütz y Aron Gurwitsch ${ }^{17}$, entre otros. Estos dos fenomenólogos fueron los primeros en identificar ciertas tesis jamesianas como propiamente fenomenológicas $y$, en ese sentido, como un anticipo, quizá menos articulado, de lo que Husserl desarrollaría con más solidez a partir de la época de las IL. La intención de las líneas que siguen es mostrar, a través de la comparación y el estudio de algunos textos de ambos autores, las razones por las cuales las afinidades entre el pensamiento de ambos se hicieron tan claras para estos discípulos de Husserl, así como para otros estudiosos. Cabe decir que, en nuestro caso, no seguiremos el hilo conductor que anima los artículos de Schütz y Gurwitsch, ya que en ambos se va bastante más allá del contexto de las IL; sin embargo, sus reflexiones se tendrán como pauta para nuestra indagación.

Quizá una de las mejores formas de seguir el rastro a esta relación intelectual sea su punto de encuentro más explícito, a saber, Franz Brentano. Tanto Husserl como James estudiaron con entusiasmo la famosa Psicología de Brentano y, de hecho, Husserl tuvo el privilegio de asistir a sus clases durante algún tiempo. Como sabemos, Husserl discute en numerosos pasajes de sus IL con Brentano y, si bien no en la misma proporción, James tiene también a Brentano, por quien tenía gran consideración, como interlocutor en varios momentos de los $P P^{18}$. Probablemente, uno de los temas que más interesó

\footnotetext{
${ }_{17}$ Para información bibliográfica más detallada, puede verse la entrada "Secondary Bibliographies. Husserl's Influence on the U.S.A.", en: The Husserl Page (consulta: 7 de julio de $2010<$ http:// www.husserlpage.com/hus_ref3.html >). Lamentablemente, en nuestro caso, solo se encuentran disponibles dos textos sobre el particular, aunque ambos de gran imporancia: Gurwitsch, A., "William James' Theory of the 'Transitive Parts' of the Stream of Consciousness", en: Philosophy and Phenomenological Research, III, No.4 (1943), pp. 449-477y Schütz, A. "William James' Concept of the Stream of Thought Phenomenologically Interpreted”, en: Philosophy and Phenomenological Research, I, No. 4 (I94I), pp. 442-452.

${ }^{18}$ Véase la nota a la p. 240 del primer tomo de los $P P$, en la cual James se refiere al autor alemán en los siguientes términos: “Altogether this chapter of Brentano's on the Unity of Consciousness
} 
a ambos autores del trabajo de Brentano fue el desarrollo inicial de lo que luego conoceríamos como el concepto de intencionalidad en Husserl. Lo interesante es que el concepto de intencionalidad se encuentra ya presente en la psicología de James, como veremos en las líneas que siguen, aunque de un modo no sistemático.

La primera vez que James desarrolló su "noción” de intencionalidad se dio en el contexto de una conferencia titulada "The Function of Cognition", ofrecida ante la Aristotelian Society el $1^{\circ}$ de diciembre de 1884 (seis años antes de la publicación de los PP) 19 . James inicia así su conferencia: "The following inquiry is [...] not an inquiry into the 'how it comes,' but into the 'what it is' of cognition. [...] Cognition is a function of consciousness. The first factor it implies is therefore a state of consciousness wherein the cognition shall take place. Having elsewhere used the word 'feeling' ${ }^{20}$ to designate generically all states of consciousness considered subjectively, or without respect to their possible function, I shall then say that, whatever elements an act of cognition may imply besides, it at least implies the existence of a feeling. [If the reader share the current antipathy to the word 'feeling,' he may substitute for it, wherever I use it, the word 'idea,' taken in the old broad Lockian sense, or he may use the clumsy phrase 'state of consciousness,' or finally he may say 'thought' instead.] Now it is to be observed that the common consent of mankind has agreed that some feelings are cognitive and some are simple facts having a subjective, or,

is as good as anything with which I am acquainted" (James, W., Principles of Psychology (I), Nueva York: Henry Holt, 1890).

${ }^{19}$ La misma fue reproducida luego en Mind, X (1885) e incluida como el primer capítulo de The Meaning of Truth en 1909. Nuestras referencias corresponden a James, W., The Meaning of Truth, en: Kuklick, B. (ed.), William James Writings, 1902-1910, Nueva York: The Library of America, 1987, séptima reimpresión, pp. 821-978.

${ }^{20}$ Es importante mencionar que Husserl se refiere también a los sentimientos en las $I L$; sin embargo, él tiene una visión muy puntual de los mismos, bastante más definida y rigurosa que la de James. Menciono esto porque, a simple vista, uno podría decir que los sentimientos son un tipo de vivencia intencional y no propiamente el equivalente a la categoría "vivencia intencional", pero en el caso de James el asunto es más amplio y es por ello que la relación sí puede establecerse. Este tema es abordado por Husserl en el §I5 de la Quinta IL y en él sostiene, en resumen, que lo sentimientos (piensa, sobre todo, en sensaciones de placer o displacer) tienden a tener carácter intencional; sin embargo, existen sentimientos (sensaciones) de naturaleza distinta, como una quemadura o una picadura de algún insecto, que no pueden ser puestos en el mismo nivel que el afecto o el desagrado. En ese sentido, Husserl concluye afirmando que no se trata del mismo género de vivencias, aunque a veces el lenguaje pueda llevarnos a confusión. CF. IL, pp. 505-509. 
what one might almost call a physical, existence, but no such self-transcendent function as would be implied in their being pieces of knowledge. Our task is again limited here. We are not to ask, 'How is self-transcendence possible?' We are only to ask, 'How comes it that common sense has assigned a number of cases in which it is assumed not only to be possible but actual? And what are the marks used by common sense to distinguish those cases from the rest?' In short, our inquiry is a chapter in descriptive psychology, -hardly anything more"21. Podríamos ir sugiriendo, a grandes rasgos, algunas cuestiones. En primer lugar, que la investigación que James se propone en torno a la cognición está centrada en lo que él llama feelings. Esta noción de sentimientos es algo general y, seguramente, no suficientemente precisa en términos fenomenológicos, pero se acerca bastante a la noción de vivencia (en sentido fenomenológico no-puro) sugerida por Husserl. En ambos casos, los autores distinguen entre la vivencia subjetiva y el acontecimiento que podríamos llamar real22. Así, para usar un ejemplo de Husserl que James compartiría, cuando una persona dice que "ha vivido una guerra", se refiere a que ha vivido los procesos externos a sí mismo que suponen un conflicto bélico; sin embargo, en términos fenomenológicos, esos procesos externos no son el contenido de la conciencia. Sus contenidos, más bien, son los procesos de percibir, juzgar, etc. referidos a aquellos. Luego, las vivencias -sentimientos, para James- son parte del torrente subjetivo, del torrente de la conciencia y, por ende, los contenidos vividos son las vivencias mismas ${ }^{23}$.

Otro asunto para destacar es la pregunta en torno a la cognición y a la posibilidad del conocimiento. El tema consiste en ver cómo es posible que se atribuya a cierto tipo de sentimientos/vivencias la capacidad de constituir conocimiento y no solo el hecho de ser experiencias inmanentes. En ese sentido, el trabajo que James se propone, como él mismo indica, es el de una psicología descriptiva que pueda dar luz sobre esta cuestión. Habría que decir

\footnotetext{
${ }^{21}$ James, W., The Meaning of Truth, p. 833; cursivas añadidas.

${ }^{22}$ Cf. ibid., p. 835, concretamente, la referencia a las "realities". James piensa que la única forma de hablar de conocimiento es que exista algo afuera que trascienda al sujeto; sin embargo, afirma que no hay garantía de que eso que se considera real lo sea de modo absoluto. Mucho de la confianza del investigador en dicha "realidad" es lo que garantiza su existencia y es posible que se trate de objetos o eventos inexistentes. Es por eso que, como veremos, en los PP dará un paso más postulando objetividades ideales en términos muy similares a los de Husserl. 
que así también entendía Husserl su trabajo en la época de la primera edición de las IL y que, de hecho, el problema del conocimiento es uno de los temas fundamentales de ese texto.

Después de varias páginas en las que James ha ido desarrollando un argumento en torno a la posibilidad del conocimiento usando como ejemplo el sentimiento de $q^{24}$, siendo $q$ cualquier evento u objeto posible distinto del sentimiento que de él se tiene, nuestro autor introduce algunas ideas que nos van acercando más a la noción de intencionalidad"25: "We the critics look on and see a real $q$ and a feeling of $q$; and because the two resemble each other, we say the one knows the other. But what right have we to say this until we know that the feeling of $q$ means to stand for or represent just that same other $q$ ? Suppose, instead of one $q$, a number of real $q$ 's in the field. If the gun shoots and hits, we can easily see which one of them it hits. But how can we distinguish which one the feeling knows? It knows the one it stands for. But which one does it stand for? It declares no intention in this respect. It merely resembles; it resembles all indifferently; and resembling, per se, is not necessarily representing or standing-for at all. Eggs resemble each other, but do not on that account represent, stand for, or know each other. And if you say this is because neither of them is a feeling, then imagine the world to consist of nothing but toothaches, which are feelings, feelings resembling each other exactly, -would they know each other the better for all that?"26

Como puede verse, se levanta aquí el problema de la adecuación entre el sentimiento de $q$ /vivencia intencional y $q /$ su objeto intencional. Se trata de un problema filosóficamente relevante, fenomenológicamente relevante, sobre todo, y Husserl se ocupa también de él en las IL. Algunas páginas después, James hace referencia ya de modo directo al carácter intencional del sentimiento: "And thus do men invariably decide such a question. The falling of the dream's practical consequences into the real world, and the extent of the resemblance

\footnotetext{
${ }^{24} \mathrm{Cf}$. James, W., The Meaning of Truth, p. 834.

${ }^{25}$ En este momento del texto, James ya ha introducido una importante diferencia entre tipos de conocimiento. Por un lado está el que denomina acquaintance or familiarity with $\mathrm{y}$, por el otro, el denominado knowledge-about. El primer caso supone solo una familiaridad con el objeto de conocimiento; el segundo, un tipo de aproximación más cercana a la de la ciencia, en tanto supone un conocimiento más exhaustivo del mismo (cf. ibid., pp. 837-840).

${ }^{26}$ Ibid., p. 840.
} 
between the two worlds are the criteria they instinctively use ${ }^{27}$. All feeling is for the sake of action, all feeling results in action, -today no argument is needed to prove these truths. But by a most singular disposition of nature which we may conceive to have been different, my feelings act upon the realities within my critic's world ${ }^{28}$. Unless, then, my critic can prove that my feeling does not 'point to' those realities which it acts upon, how can he continue to doubt that he and I are alike cognizant of one and the same real world? If the action is performed in one world, that must be the world the feeling intends; if in another world, that is the world the feeling has in mind. If your feeling bear no fruits in my world, I call it utterly detached from my world; I call it a solipsism, and call its world a dream-world. If your toothache do not prompt you to act as if I had a toothache, nor even as if I had a separate existence; if you neither say to me, 'I know now how you must suffer!' nor tell me of a remedy, I deny that your feeling, however it may resemble mine, is really cognizant of mine. It gives no sign of being cognizant, and such a sign is absolutely necessary to my admission that it is" 29 .

Como puede verse con claridad, James da al sentimiento la estructura intencional que Husserl otorga a las vivencias y lo hace con un vocabulario no tan lejano al del autor alemán ${ }^{30}$. Es evidente que James no tiene el cuidado y el ánimo de precisión que sí ofrece Husserl, pero eso se debe, entre otras cosas, a que James no tenía pretensiones del mismo tipo que las del autor

\footnotetext{
${ }^{27}$ Antes del establecimiento del mencionado criterio, James había colocado un ejemplo donde se hacía compleja la diferenciación entre realidad y el sueño.

${ }^{28}$ El mundo de los críticos es aquel del psicólogo descriptivo que está analizando el proceso cognitivo.

${ }^{29}$ Ibid., pp. 842-843.

${ }^{30}$ Nuevas menciones del carácter intencional de los sentimientos pueden encontrarse más adelante, cf. ibid., pp. 846 (nota I), 847, 849, 850, así como en las consideraciones finales, sobre todo en las 4 y 5 . Cabe destacar que, en este mismo texto, James introduce también la distinción husserliana entre vivencias significativas y vivencias intuitivas o plenas. La misma puede verse, siempre con menos nivel de detalle, en su ejemplo sobre la oración 'Newton saw the handiwork of God in the heavens as plainly as Paley in the animal kingdom'. El argumento que está detrás es que existen distintos tipos de sentimientos/vivencias y que no todas suponen una intuición actual del objeto en cuestión (Newton, Payley, God, etc.) y que, sin embargo, dichos sentimientos no dejan por ello de tener sentido ni de ser capaces de aportar conocimiento (cf. ibid., p. 848ss). Esta misma distinción puede encontrarse en textos posteriores, como el siguiente de los PP: "To speak in technical language, a subject may be conceived by its denotation, with no connotation, or a very minimum of connotation, attached. The essential point is that it should be re-identified by us as that which the talk is about; and no full representation of it is necessary for this, even when it is a fully representable thing" (PP, p. 463).
} 
de las IL. Sin embargo, e independientemente de que uno esté de acuerdo o no con el resto del argumento, hay aquí importantes elementos de juicio que perduraron en los PP y que, probablemente, tuvieron una importante influencia en Husserl. Cabe notar, como elemento añadido, que ya desde esta época pueden advertirse, como se ve en la cita, algunas de las razones que llevaron a James a desarrollar más adelante su pragmatismo y a enfatizar en él el rol de la acción como criterio dirimente en torno a la justificación de las creencias ${ }^{31}$.

Si bien, a partir de los fragmentos citados, alcanzamos a identificar algunos puntos de contacto, es propiamente en el contexto de los PP donde podemos encontrar las conexiones más explícitas, las mismas que se intensifican si recordamos la atenta lectura que hizo Husserl de esta obra. Existen varios caminos que nos pueden permitir examinar dichos vínculos, pero creo que el capítulo XII del texto, "Conception”, puede ser nuestra mejor puerta de entrada.

Lo primero que habría que indicar es que James, para este momento del libro, ya ha introducido su célebre noción de la corriente del pensamiento (PP, XIX, "The Stream of Thought"). La misma, de modo muy escueto, puede quedar resumida en la indicación que el propio James inserta al inicio del capítulo del mismo nombre. Así, nuestro autor señala que el pensamiento tiene cinco características principales: “I) Every thought tends to be part of a personal consciousness. 2) Within each personal consciousness thought is always changing. 3) Within each personal consciousness thought is sensibly continuous. 4) It always appears to deal with objects independent of itself. 5) It is interested in some parts of these objects to the exclusion of others, and welcomes or rejects -chooses from among them, in a word-all the while" ${ }^{32}$. Es importante resaltar estas cuestiones por varias razones. En primer lugar, hay que indicar

31 "Desde luego, las disputas que se suscitan no tienen fin, y en tales casos el método pragmático trata de interpretar cada una de esas ideas señalando sus respectivas consecuencias prácticas. ¿Qué diferencia de orden práctico supondría para alguien el que fuera verdadera tal idea en vez de su contraria? Si no puede señalarse ninguna diferencia práctica, entonces las alternativas significan lo mismo de manera práctica, y toda su disputa es vana. Cuando una disputa es seria, debemos ser capaces de mostrar alguna diferencia práctica que tiene que seguirse [de] si un lado o el otro está en lo cierto" (James, W., Pragmatismo. Un nuevo nombre para viejas formas de pensar, traducción de Ramón del Castillo, Madrid: Alianza Editorial, 2000, p. 80).

32 PP, p. 225. 
que la idea del stream of thought ${ }^{33}$ parece haber tenido notoria influencia en Husserl, como se atestigua ya desde las $I L^{34}$; pero se trata de una influencia que sobre todo se nota en los análisis posteriores del filósofo alemán. Otro asunto interesante, aunque solo lo menciono, se nota cuando uno repara en las similitudes que se pueden hallar entre los desarrollos jamesianos del punto 5 , su teoría de los fringes, y la noción de horizonte en Husserl ${ }^{35}$.

Más allá de estos comentarios, la cuestión de la corriente o del torrente de pensamiento es importante porque esta le permite a James plantear un significativo problema teórico que desarrollará con detenimiento en el capítulo sobre la concepción. El problema puede plantearse en los siguientes términos: "Are not the sensations we get from the same object, for example, always the same? Does not the same piano-key, struck with the same force, make us hear in the same way? Does not the same grass give us the same feeling of green, the same sky the same feeling of blue, and do we not get the same olfactory sensation no matter how many times we put our nose to the same flask of cologne? It seems a piece of metaphysical sophistry to suggest that we do not; and yet a close attention to the matter shows that there is no proof that the same bodily sensation is ever got by us twice" ${ }^{36}$. El asunto que preocupa a James es el de la posibilidad del conocimiento, de allí la pertinencia de nuestra inicial referencia al asunto a través de "The Function of Cognition". Si nunca tenemos una vivencia idéntica, aunque sea del mismo objeto, icómo es posible que pretendamos conocer o, más aun, conocer algo común a otras personas? James desliza una respuesta inicial a ese problema en la línea que sigue al fragmento citado: "What is got twice is the same object"37; sin embargo, las

\footnotetext{
${ }^{33}$ James se refiere así a este concepto: "Consciousness, then, does not appear to itself chopped up in bits. Such words as 'chain' or 'train' do not describe it fitly as it presents itself in the first instance. It is nothing jointed; if flows. A 'river' or a 'stream' are the metaphors by which it is most naturally described. In talking of it hereafter, let us call it the stream of thought, of consciousness, or of subjective life" (ibid., p. 239).

${ }^{34}$ Véase, por ejemplo: "[...] ciertos contenidos son componentes en una unidad de conciencia, en la corriente fenomenológicamente unitaria de la conciencia [...]" (IL, p. 479; énfasis añadido). ${ }^{35}$ En general, cf. Husserl, E., Ideas relativas a una fenomenología pura y una filosofía fenomenológica, traducción de José Gaos, 2da. edición, México D.F./Buenos Aires: Fondo de Cultura Económica, 1962. El tema del los horizontes es desarrollado por Herzog, M., op. cit., pp. 39-40. Las relaciones generales entre el stream of thought y la fenomenología de Husserl están bien trabajadas en Schütz, A., op. cit., p. 446ss.

${ }^{36}$ PP, p. 231. 
sugerencias más interesantes se encuentran más adelante, en el capítulo del cual nos ocuparemos de inmediato.

James inicia el capítulo XII de los PP ampliando la respuesta que acabamos de enunciar: "The possibility of two such knowledges ${ }^{38}$ depends on a fundamental psychical peculiarity which may be entitled 'the principle of constancy in the mind's meanings', and which may be thus expressed: 'The same matters can be thought of in successive portions of the mental stream, and some of these portions can know that they mean the same matters which the other portions meant'. One might put it otherwise by saying that 'the mind can always intend, and know when it intends, to think of the Same"”39. Así, James aborda el problema del flujo perpetuo de la conciencia apelando a una característica peculiar de nuestra psique, a saber, la posibilidad de tener significados constantes sin que esto suponga que la corriente del pensamiento se detenga. El tema de la intencionalidad está claramente indicado aquí, la idea es que nuestra conciencia puede referirse a un determinado objeto, apuntar hacia él, tenerlo como objeto intencional, en diferentes momentos del fluir del pensamiento ${ }^{40}$.

James amplía este asunto al referirse a este principio de los significados constantes con el nombre de "the sense of sameness" y precisa de inmediato que: "[...] we are in the first instance speaking of the sense of sameness from the point of view of the mind's structure alone, and not from the point of view of the universe. We are psychologizing, not philosophizing. That is, we do not care whether there be any real sameness in things or not, or whether the mind be true or false in its assumptions of it. Our principle only lays it down that the mind makes continual use of the notion of sameness, and if deprived of it, would have a different structure from what it has. In a word, the principle that the mind can mean the Same is true of its meanings, but not necessarily of aught besides"4l. Esta sensación de igualdad es la que, previa a todo juicio sobre el mundo exterior, nos ofrece un primer esbozo de que hay algo más

\footnotetext{
${ }^{38}$ Se refiere a los ya mencionados "bare acquaintance with them and knowledge about them".

${ }^{39}$ Ibid., p. 459.

${ }^{40}$ Husserl precisa que cuando se refiere al adjetivo intencional, este "indica el carácter esencial común al tipo de vivencias que se trata de definir, la propiedad de la intención, el referirse a algo objetivo en el modo de la representación o en cualquier modo análogo" (IL, p. 498).

${ }^{41}$ PP, pp. 459-460.
} 
aparte del mero fluir de nuestra conciencia ${ }^{42}$; sin embargo, se trata solo de un esbozo, de una sensación que podría dejarnos, como sugiere la cita, en una situación solipsista que tanto Husserl como James rechazarían. Es cierto que la entrada de James, como él mismo indica, es psicológico-descriptiva, y lo mismo sucede con Husserl en las IL, pero eso no parece bastar como excusa para responder al aparente problema de la inmanencia psicológica frente al que nos encontramos. Sobre este asunto volveremos de inmediato, pero me interesa que nos dirijamos brevemente a las IL para que notemos el gran nivel de afinidad que existe entre ese texto; me refiero concretamente al §I4 de la Quinta Investigación y los pasajes de los PP que venimos citando.

En el parágrafo mencionado, Husserl se hace cargo de algunas de las objeciones que le han sido presentadas en torno a su noción de vivencia intencional que, como hemos dicho, guarda gran semejanza con la noción de sentimiento en James y también con sus desarrollos posteriores en torno a la concepción. Una de las principales figuras críticas es Natorp; sin embargo, aduce Husserl, buena parte de las reservas provienen de extravíos producidos por la propuesta de Brentano y no tanto por la suya. Independientemente de a quién corresponda la crítica de Natorp, Husserl recoge un ejemplo de este último relativo a la audición y, si bien está de acuerdo con el mismo, no ve que en él se halle razón para cuestionar la delimitación de su noción de vivencia intencional. Natorp sostiene que no es posible considerar el propio oír sin pensar en el sonido oído, tratando de decir con eso que se trata de dos experiencias indesligables cuando se las analiza en el fuero de la primera persona. Husserl está de acuerdo, pero no ve cómo esa precisión afecta su propia delimitación, ya que aún pueden distinguirse dos esferas: "el sonido oído (el objeto de la percepción) y el oír el sonido (el acto de percepción)”43. O puesto en la terminología de "The Function of Cognition", q y el sentimiento de $q$. Lo que resulta evidente para Husserl es que se trata de dos esferas fenomenológicamente diferenciables, aunque articuladas, y eso es lo relevante para su investigación, así como para la de James.

42 "The first spaces, times, things, qualities, experienced by the child probably appear [...] as simple beings, neither in nor out of thought. But later, by having other thoughts than this present one, and making repeated judgments of sameness among their objects, he corroborates in himself the notion of realities, past and distant as well as present" (ibid., p. 272). 
Sin embargo, Husserl nos propone seguir analizando el caso de la audición planteado por Natorp. "Yo oigo puede significar en psicología yo tengo una sensación; en el lenguaje usual significa yo percibo: yo oigo el adagio del violín, el trinar de los pájaros, etc. Distintos actos pueden percibir lo mismo y, sin embargo, implicar sensaciones totalmente diversas" ${ }^{\prime 4}$. Podemos oír el mismo sonido, pero las características que rodean la experiencia pueden determinar sensaciones disímiles según el sonido esté a mayor o menor distancia, por ejemplo. No solo eso, sino que es posible que, teniendo contenidos de sensación iguales en la conciencia, percibamos distintos objetos. En todo caso, indica Husserl, ya cuando hablamos de aprehensión o de percepción del objeto correspondiente el asunto no puede quedar reducido a una afluencia de sensaciones: hablamos allí de un “'modo de la conciencia', un estado del espíritu”45. Este es el mismo problema que ocupa a James, a saber, el de poder establecer una adecuada relación de correspondencia entre vivencia y objeto. El asunto, en su caso, será ampliado con más detalle cuando se examine el carácter funcional de nuestras concepciones, pero cabe mencionar ya que su propuesta también conducirá a plantear relaciones ideales de concepción como sucede, aunque en su propio lenguaje, con Husserl. De hecho, es el mismo autor alemán quien indica, poco después de los pasajes que venimos comentando, que ese breve examen puede entregarnos "su contenido fenomenológico puro, si eliminamos todo lo real-empírico": nos permitirá ver con el intelecto, "como situación general esencial, que el ser del contenido sentido es muy distinto del ser del objeto percibido, el cual es presentado por el contenido, pero no es consciente realmente" ${ }^{\prime \prime 6}$. Si bien James no fue nunca muy dado al lenguaje "fenomenológico puro", es claro que él también notó la necesidad racional de establecer idealmente las mencionadas distinciones esenciales con la finalidad de no quedar sumergidos en un psicologismo que, como bien denunciaba Husserl, no conduciría más que al escepticismo.

Ahora bien, sigamos con el texto de Husserl para ver cómo la idea del sense of sameness es abordada también por este filósofo y en términos muy cercanos a los de William James. Husserl, después de examinar la crítica de Natorp, decide ahondar en el asunto, pero utilizando ejemplos visuales y ya no auditivos. Nos

\footnotetext{
${ }^{44} \mathrm{lbid}$.

45 Ibid., p. 50 I.

${ }^{46} \mathrm{lbid}$.
} 
pide, entonces, que pongamos un objeto cualquiera ante nuestros ojos y nos invita luego a realizar el análisis. Resulta claro que tenemos siempre el mismo "contenido de conciencia", si es que así denominamos al objeto percibido, un tigre de la India, para usar un ejemplo de James que Husserl conocía bien ${ }^{47}$. En cambio, podemos decir que tenemos nuevos contenidos de conciencia si nos referimos a los contenidos vividos: la visión por escorzos de ese mismo tigre dependiendo del ángulo desde el cual se lo mire, la visión del tigre diez años después de nuestro primer encuentro, etc. Son vividos diversos contenidos, pero el objeto percibido es siempre el mismo. Así, en sentido estricto, el contenido vivido no es el objeto percibido. Añádase que el hecho de que el objeto percibido exista o no es algo irrelevante: podría tratarse de ese tigre de la India real o del dios Júpiter mencionado por Husserl algunas páginas antes, para el caso da lo mismo. Finalmente, es inherente a la vivencia el hecho de que creamos aprehender un mismo objeto a pesar de los diversos contenidos vividos. Es lo que Husserl llama conciencia de identidad y es, a su vez, lo que James llamaba the sense of sameness, la noción que lo conduciría a él también, algunos años antes que a Husserl, a hablar de la necesidad de objetividades ideales o, más precisamente, de la unidad ideal de la especie.

Habiendo ampliado con algo más de detalle las conexiones entre los análisis de ambos autores, quisiera finalizar este trabajo regresando sobre el texto de los PP para mostrar con algo más de evidencia textual el camino que lleva a James a sostener, en sus propios términos, dicha unidad ideal de la especie. En ese sentido, el modo en que James comprende la concepción puede ayudarnos a esclarecer un poco el problema: "The function by which we thus identify a numerically distinct and permanent subject of disclosure is called CONCEPTION; and the thoughts which are its vehicles are called concepts. [...] The word 'conception' is unambiguous. It properly denotes neither the mental state nor what the mental state signifies, but the relation between the two, namely, the function of the mental state in signifying just that particular thing"48. Como puede verse, la noción de concepción que James maneja es muy cercana a la que Husserl mantiene en relación a la intencionalidad, pero lo más notable del carácter funcional de nuestras concepciones es que estas ponen de 
manifiesto el vínculo entre inmanencia y trascendencia, entre sentimientos y mundo exterior, para usar el lenguaje de James ${ }^{49}$. En ese sentido, Herzog indica que: “James's 'concept' is, like Husserl's notion of intentionality, neutral with respect to the ontological question of the reality of things. Conception in James, and intentionality in Husserl, are both determined by the function of a state of consciousness in which a something is always something specific, the connection of conscious lived experience to something" 50 .

Ahora bien, la noción de concepción que James sostiene resulta relevante porque, como mencionamos líneas atrás, the sense of sameness nos muestra cómo existe una función psíquica que nos permite identificar continuidades en el medio del stream of thought; sin embargo, se trataba, hasta donde vimos, de una continuidad descriptible en el solo fluir de la conciencia y no, aún, más allá de ella. Por ello, James mismo concede que hace falta avanzar un poco más para esclarecer el problema y establecer una conexión de modo más completo: "Confusion occurs when we do not know whether a certain object proposed to us is the same with one of our meanings or not; so that the conceptual function requires, to be complete, that the thought should not only say 'I mean this,' but also say 'I don't mean that"” ${ }^{51}$. Luego, cuando uno está en capacidad de determinar con claridad la relación entre sentimiento de $q$ /vivencia intencional y q/objeto intencional, el acto de concepción se ha realizado con propiedad. Lo más interesante, no obstante, es que es evidente que desde una perspectiva psicologista, en el sentido husserliano, esta función conceptual no podría acontecer en el sentido que sugiere James, ya que para un psicologista el papel funcional de nuestras concepciones no podría tener garantía en vista de que la relación entre vivencia y objeto intencionales sería meramente empírica ${ }^{52}$. En ese sentido, el aporte de James $-y$ el notable

\footnotetext{
${ }^{49}$ Cabe recordar que hablamos aquí con un lenguaje que puede resultar equívoco. En los PP, James trata de presentar distinciones analíticas con la finalidad de hacer un análisis descriptivo de nuestros procesos psicológicos; sin embargo, y esto se nota con más fuerza en una obra como los Essays in Radical Empiricism, nuestro autor era enemigo de toda posición dualista, de las teorías que consideraban a la mente como un escenario completamente independiente del resto de la naturaleza, etc. Por estas razones, James desarrolló su concepto de pure experience, aunque eso le trajo también otros problemas. Cf. Embree, L. y otros (eds.), op. cit., p. 363.

${ }^{50}$ Herzog, M., op. cit., p. 36.

${ }^{51}$ PP, pp. 46I-462.

${ }^{52} \mathrm{Al}$ respecto, indica Cobb-Stevens: "If meanings cannot be reduced to blurred general images, then neither can logical laws be reduced to inductive generalizations about our thought processes. James criticizes John Stuart Mill (1806-1873) and Herbert Spencer (I820-1903) for
} 
desarrollo de Husserl- consiste en la postulación de objetividades ideales, las mismas que sin existir en coordenadas espacio-temporales constituyen la condición de posibilidad de la capacidad cognoscitiva, de la posibilidad misma del conocimiento que tanto preocupaba a nuestros dos autores. Veamos cuál es el aporte de James a este respecto.

El siguiente pasaje puede parecer sorprendente en un autor como James, más conocido en el terreno de la filosofía por su pragmatismo que por su psicología descriptiva de los procesos de la conciencia; no obstante, arroja mucha luz sobre el problema que venimos examinando: "Each conception thus eternally remains what it is, and never can become another. The mind may change its states, and its meanings, at different times; may drop one conception and take up another, but the dropped conception can in no intelligible sense be said to change into its successor. The paper, a moment ago white, I may now see to have been scorched black. But my conception 'white' does not change into my conception 'black'. On the contrary, it stays alongside of the objective blackness, as a different meaning in my mind, and by so doing lets me judge the blackness as the paper's change. Unless it stayed, I should simply say 'blackness' and know no more. Thus, amid the flux of opinions and of physical things, the world of conceptions, or things intended to be thought about, stands stiff and immutable, like Plato's Realm of Ideas"53. En efecto, el pasaje llama la atención, aunque si uno se mantiene atento a la reflexión que James venía realizando, incluso antes de los PP, puede notar que se trata, más bien, de una propuesta bastante consecuente. A su vez, el lector de Husserl puede notar con algo más de claridad cómo James influyó positivamente en el trabajo del autor alemán. Recordemos que ya habíamos hecho mención de que Husserl consideraba a James como un valioso predecesor de sus propios estudios y, más importante, como un filósofo cuyos análisis lo habían alejado poderosamente de cualquier influencia psicologista. La referencia a la idealidad de las concepciones, e incluso esta mención de un cierto platonismo, hacen notorio el nivel de influencia del autor estadounidense. En palabras de

failing to account for the fact that the logical network of our meanings forms an ideal system whose relations have a necessity and universality that cannot be accounted for by habit and association" (Embree, L. y otros (eds.), op. cit., p. 364).

${ }^{53}$ PP, p. 462; cursivas finales añadidas. Cabe recordar que Husserl admitía para sí mismo un cierto tipo de platonismo en relación a las objetividades ideales, aunque sin conceder a estas densidad ontológica, como sí sucedía con el filósofo griego. 
Herzog, "James undermines the antagonism of empiricism and apriorism with his doctrine of conceptions, and in this shows himself to be the epistemological precursor of phenomenology" 54 .

\section{§ 3. Corolario}

Después de todo lo visto hasta aquí, creo que podemos sostener sin demasiada dificultad que existen argumentos suficientes para considerar que los PP de William James tuvieron una importante influencia en las IL de Edmund Husserl e incluso en textos posteriores como Ideas relativas a una fenomenología pura y una filosofía fenomenológica. Dicha influencia es declarada por el mismo Husserl en comunicaciones personales y en sus propias notas, además de identificable a través de las obras de James que Husserl leyó con detenimiento, subrayó y anotó copiosamente.

Independientemente de eso, la posibilidad de determinar el nivel de influencia de James en Husserl es siempre relativa. Si bien sabemos que esta existió, aventurarnos a decir que fue determinante, insustituible o algo por el estilo sería siempre afirmar demasiado. El hecho es que Husserl desarrolló una reflexión autónoma y que si bien mantiene varios motivos comunes con James, su aproximación pudo sin problema haber surgido de modo independiente. Además, es obvio, como ya se indicó, que la cercanía de ambos autores no fue perenne y que el giro trascendental de la fenomenología husserliana alejó al filósofo alemán de los supuestos filosóficos de James ${ }^{55}$, aunque quizá no tanto de su metodología de análisis.

Sea como fuere, lo que este trabajo ha querido mostrar es que hay razones suficientes para sostener la sintonía de las reflexiones de ambos autores. La misma se hace evidente en torno al concepto de intencionalidad y a los alcances del mismo; sin embargo, se han podido elegir entradas algo distintas, como,

\footnotetext{
${ }^{54}$ Herzog, M., op. cit., p. 37.

${ }^{55}$ Aunque cabe indicar aquí que el llamado giro trascendental no supone, si se mira con cuidado, una ruptura entre las ideas del Husserl temprano y aquellas desarrolladas por este autor en su época de madurez. Es más preciso hablar de una profundización y radicalización de aquellas intuiciones básicas que, sin embargo, estuvieron permanentemente presentes en el pensamiento del filósofo alemán.
} 
por ejemplo, la de la percepción del yo o la de la percepción del tiempo, entre otras. Este trabajo muestra, además, que la relación entre ambos pensadores constituye una senda de investigación no solo interesante, sino filosóficamente provechosa, que, además, supone una tarea pendiente en lengua española. Hay alguna importante información recabada en inglés y en alemán en torno a los vínculos entre estos filósofos, pero un trabajo más detenido sobre ellos podría arrojar luz sobre la influencia histórica que tanto el pragmatismo como la fenomenología han tenido en la reflexión contemporánea, particularmente en hispanoamérica. Existen ya en lengua hispana algunos trabajos que estudian a Husserl y a James de modo independiente no solo en lo referido a sus respectivas aproximaciones filosóficas, sino también en lo relativo a su influencia en América Latina ${ }^{56}$; sin embargo, una mirada de conjunto que sistematice la información ya existente es, creo yo, una valiosa tarea pendiente.

${ }^{56}$ A este respecto, por lo menos dos textos merecen mención. En la senda del pragmatismo: Quintanilla, P., C. Escajadillo y R. Orozco, Pensamiento y acción. La filosofía peruana a comienzos del siglo XX, Lima: Instituto Riva-Agüero/Fondo Editorial de la PUCP, 2009; por el lado de los estudios fenomenológicos: Begué, M.-F., Fenomenología en América Latina, Bogotá: Universidad de San Buenaventura, 2000. 